\title{
Modulation of ion and electron pitch angle in the presence of large-amplitude, low-frequency, left-hand circularly polarized electromagnetic waves observed by MMS
}

\author{
J. S. Zhao, T. Y. Wang, M. W. Dunlop, J. S. He, X. C. Dong, \\ D. J. Wu, Yu. V. Khotyaintsev, R. E. Ergun, C. T. Russell, B. L. Giles, \\ R. B. Torbert, and J. L. Burch
}

\section{Published version information}

Citation: JS Zhao et al. "Modulation of ion and electron pitch angle in the presence of large-amplitude, low-frequency, left-hand circularly polarized electromagnetic waves observed by MMS." Astrophysical Journal, vol. 867, no. 1 (2018): 58.

DOI: $10.3847 / 1538-4357 / a a e 097$

This version is made available in accordance with publisher policies. Please cite only the published version using the reference above. This is the citation assigned by the publisher at the time of issuing the APV. Please check the publisher's website for any updates. 


\title{
Modulation of Ion and Electron Pitch Angle in the Presence of Large-amplitude, Low-frequency, Left-hand Circularly Polarized Electromagnetic Waves Observed by $M M S$
}

\author{
J. S. Zhao ${ }^{1,2}$ (1) T. Y. Wang ${ }^{2}$, M. W. Dunlop ${ }^{2,3}$, J. S. He ${ }^{4}$, X. C. Dong ${ }^{3}$, D. J. Wu ${ }^{1}$, Yu. V. Khotyaintsev ${ }^{5}$, R. E. Ergun ${ }^{6}$, \\ C. T. Russell ${ }^{7}$, B. L. Giles ${ }^{8}$, R. B. Torbert ${ }^{9}$, and J. L. Burch ${ }^{10}$ \\ ${ }^{1}$ Key Laboratory of Planetary Sciences, Purple Mountain Observatory, Chinese Academy of Sciences, Nanjing 210008, People's Republic of China \\ ${ }^{2}$ RAL Space, STFC, Oxfordshire, OX11 0QX, UK \\ ${ }_{4}^{3}$ School of Space and Environment, Beihang University, Beijing 100191, People's Republic of China \\ ${ }^{4}$ School of Earth and Space Sciences, Peking University, Beijing 100871, People's Republic of China \\ ${ }^{5}$ Swedish Institute of Space Physics, Uppsala SE-75121, Sweden \\ ${ }^{6}$ Laboratory of Atmospheric and Space Physics, University of Colorado, Boulder, Colorado 80303, USA \\ ${ }^{7}$ Institute of Geophysics and Planetary Physics, University of California, Los Angeles, CA, USA \\ ${ }^{8}$ NASA Goddard Space Flight Center, Greenbelt, MD 20771, USA \\ ${ }^{9}$ University of New Hampshire, Durham, New Hampshire, USA \\ ${ }^{10}$ Southwest Research Institute, San Antonio, Texas 78238, USA \\ Received 2018 July 26; revised 2018 August 24; accepted 2018 September 9; published 2018 October 30
}

\begin{abstract}
Most studies on low-frequency electromagnetic cyclotron waves have assumed a small wave amplitude, which ensures the reasonable application of linear and quasi-linear theories. However, the topic of large-amplitude electromagnetic cyclotron waves has not received much attention. Using Magnetospheric Multiscale measurements, this study observes low-frequency, left-hand circularly polarized electromagnetic waves with magnetic fluctuation $\sim 1-2 \mathrm{nT}$ in the dusk flank side of the Earth's magnetosheath. Considering the ambient magnetic field $\sim 15 \mathrm{nT}$ therein, the relative wave amplitude is of the order of 0.1 . These large magnetic field fluctuations result in a periodic variation of the ion pitch angle. The electron pitch angle exhibits a localized distribution feature with a timescale approximating the wave period. Moreover, some electrons are trapped at a pitch angle $\sim 90^{\circ}$, and the trapping is more remarkable as strong waves arise. These two features of the electron pitch angle distribution imply that the trapping of electrons (partly) results from large-amplitude electromagnetic cyclotron fluctuations. Our results illustrate the important role of large-amplitude electromagnetic cyclotron waves on the dynamics of charged particles.
\end{abstract}

Key words: instabilities - plasmas - waves

\section{Introduction}

Low-frequency electromagnetic cyclotron waves (i.e., ion cyclotron waves) are usually observed in different solarterrestrial environments, i.e., the Earth's magnetosphere, the magnetosheath, and the solar wind (e.g., Anderson \& Fuselier 1993, 1994; Dunlop et al. 2002; Jian et al. 2014; Remya et al. 2014; Wicks et al. 2016; Zhao et al. 2018). These waves play an important role in the dynamics of charged particles. Linear and quasi-linear theories have shown that the resonance interaction of charged particles with ion cyclotron waves will result in the loss of relativistic electrons in the Earth's radiation belt (e.g., Summers \& Thorne 2003; Summers et al. 2007; Jordanova et al. 2008) and the precipitation of ring current ions (e.g., Jordanova et al. 2001). Nonlinear mechanisms, including phase bunching and phase trapping of ions by ion cyclotron waves, can lead to a change in the ion pitch angle (e.g., Bortnik et al. 2010; Omidi et al. 2010; Zhu et al. 2012), i.e., the enhancement of low-energy protons and helium particles at the pitch angle $\sim 90^{\circ}$ observed by the AST-6, GOES1 and 2, and AMPTE/CCE spacecraft (e.g., Mauk et al. 1981; Roux et al. 1982; Anderson \& Fuselier 1994). However, in comparison with studies relating to small-amplitude waves, research on large-amplitude, low-frequency electromagnetic cyclotron waves has not attracted much attention.

Large-amplitude, circularly polarized electromagnetic proton cyclotron waves have been observed in the Earth's dusk-flank magnetosheath by Wind and Cassini measurements (Tsurutani et al. 2002; Remya et al. 2014), which find the magnetic field fluctuation to be $\sim 14 \mathrm{nT}$ in the ambient magnetic field of 〜55 nT. Using Magnetospheric Multiscale (MMS; Burch et al. 2016) measurements, this study provides other observational evidence for the existence of large-amplitude, lowfrequency electromagnetic cyclotron waves in the dusk-flank side of the Earth's magnetosheath. We show that the wave amplitude is about $1-2 \mathrm{nT}$, where the ambient magnetic field is $\sim 15 \mathrm{nT}$. The relative amplitude is of the order of 0.1 . Since the plasma measurement in the $M M S$ has high time resolution, i.e., $0.15 \mathrm{~s}$ for ions and $0.03 \mathrm{~s}$ for electrons, it provides an excellent opportunity to study ion and electron dynamics in the presence of large-amplitude electromagnetic waves.

Since we investigate both wave and particle behavior, we analyze $M M S$ data from instruments including electric field double probes (Ergun et al. 2016; Lindqvist et al. 2016), fast plasma investigation (FPI; Pollock et al. 2016), and fluxgate magnetometers (Russell et al. 2016).

\section{Observations}

Figure 1 shows an overview of the observed wave during the time interval from 14:00:00 UT to 14:06:00UT on 2017 October 7. The $M M S$ is located at nearly $(-3.9,23.7,5.7)$ Earth radii in Geocentric Solar Magnetospheric coordinates, corresponding to the dusk-flank magnetosheath. The magnetic fluctuations are 


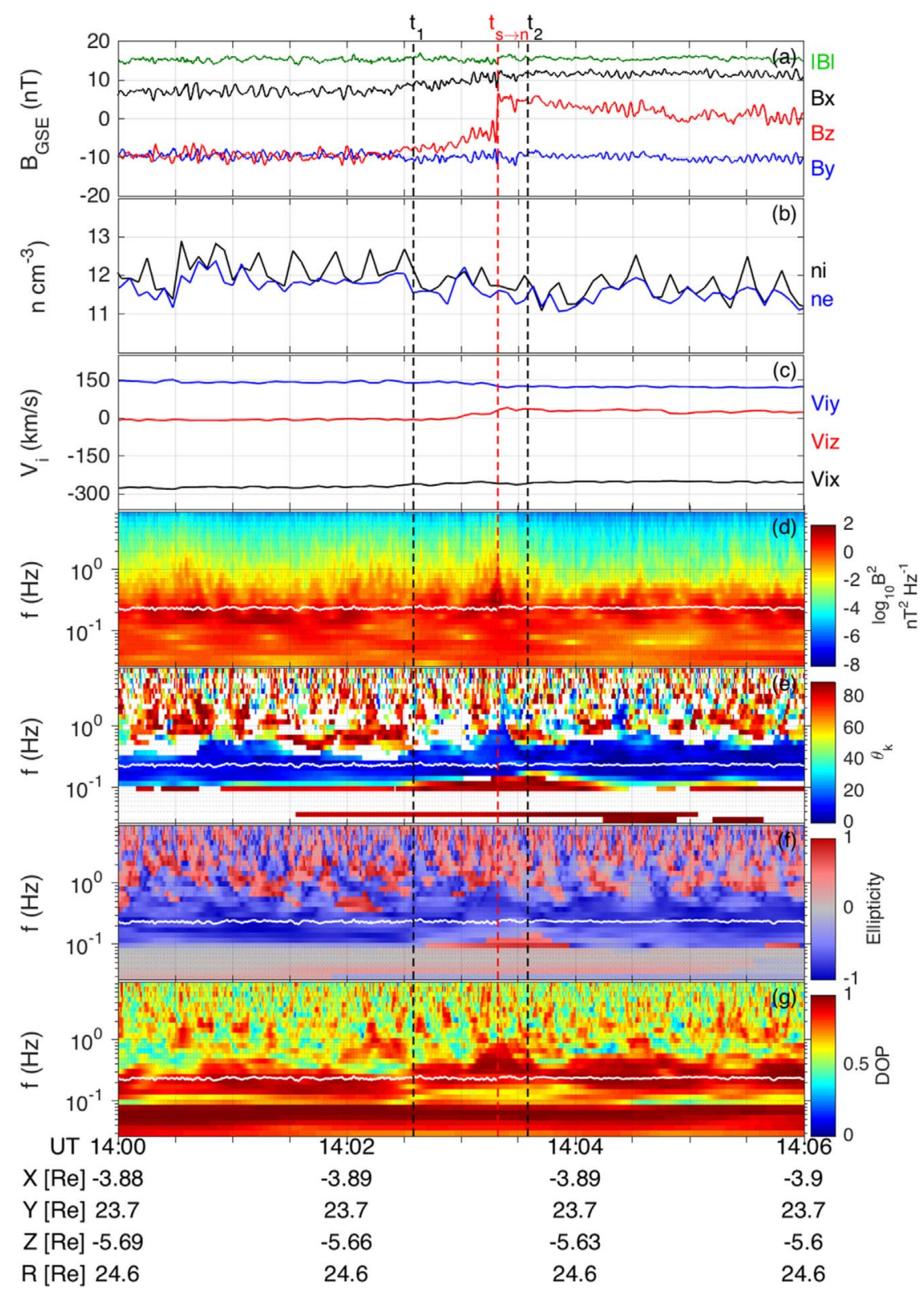

Figure 1. $M M S$ overview of the low-frequency electromagnetic cyclotron wave from 14:00:00 to 14:06:00 UT on 2017 October 7. (a) Magnetic field in Geocentric Solar Ecliptic coordinates, (b) ion and electron density, (c) ion velocity, (d) power spectral density of the magnetic field, (e) wave normal angle, (f) wave ellipticity, and $(\mathrm{g})$ degree of polarization. The solid lines in panels (d)- $(\mathrm{g})$ denote the proton cyclotron frequency. $t_{s \rightarrow n}$ corresponds to the time when the direction of $B_{z}$ changes from southward to northward. Brst data in the time interval $\left[t_{1} t_{2}\right]$ is analyzed in Figures 3-4.

distinct over the whole time interval. At $t_{s \rightarrow n} \sim 14: 03: 37.37 \mathrm{UT}$, which is denoted by the red vertical dashed line in Figure 1, the direction of $B_{z}$ changes from southward to northward. The plasma density and ion streaming velocity are relatively steady. The averaged velocities, i.e., $V_{i x} \sim-265 \mathrm{~km} \mathrm{~s}^{-1}, V_{i y} \sim 124 \mathrm{~km} \mathrm{~s}^{-1}$, and $V_{i z} \sim 16 \mathrm{~km} \mathrm{~s}^{-1}$, indicate ions streaming anti-parallel to the magnetic field. Polarization analysis in panels (d) $-(\mathrm{g})$ show that the magnetic fluctuations in the frequency range $\sim[0.1,0.5] \mathrm{Hz}$ have the following properties: (1) degree of polarization larger than 0.8 ; (2) ellipticity smaller than -0.8 ; (3) the normal angle smaller than $20^{\circ}$ or larger than $160^{\circ}$. A $180^{\circ}$ uncertainty for the normal angle comes from the wavelet (Morlet) analysis used in panel (e). Because of the inverse Poynting flux (not shown in Figure 1), and the positive correlation between velocity and magnetic fluctuations (shown in Figure 2), we conclude the normal angle is larger than $160^{\circ}$. This wave information indicates that the mode corresponds to a nearly anti-parallel propagating, left-hand circularly polarized wave, usually called the electromagnetic ion cyclotron wave in studies of the planetary magnetosphere.

Figure 2 gives electromagnetic and velocity fluctuations in field-aligned coordinates. Since an abrupt change of the magnetic field configuration, not the wave fluctuation, occurs at nearly $t_{s \rightarrow n}$, we choose the time interval of 14:02:35.00UT14:03:17.00UT before $t_{s \rightarrow n}$ to explore the general properties of the electromagnetic and velocity fluctuations. Full and filtered magnetic field components are shown in panels (a) and (b). Referring to Figure 1, coherent waves concentrate in the frequency range $0.1 \mathrm{~Hz} \leqslant f \leqslant 0.5 \mathrm{~Hz}$; therefore, this frequency range is selected as the filtered frequency. The magnetic fluctuation is of the order of $1 \mathrm{nT}$, and the largest amplitude can 

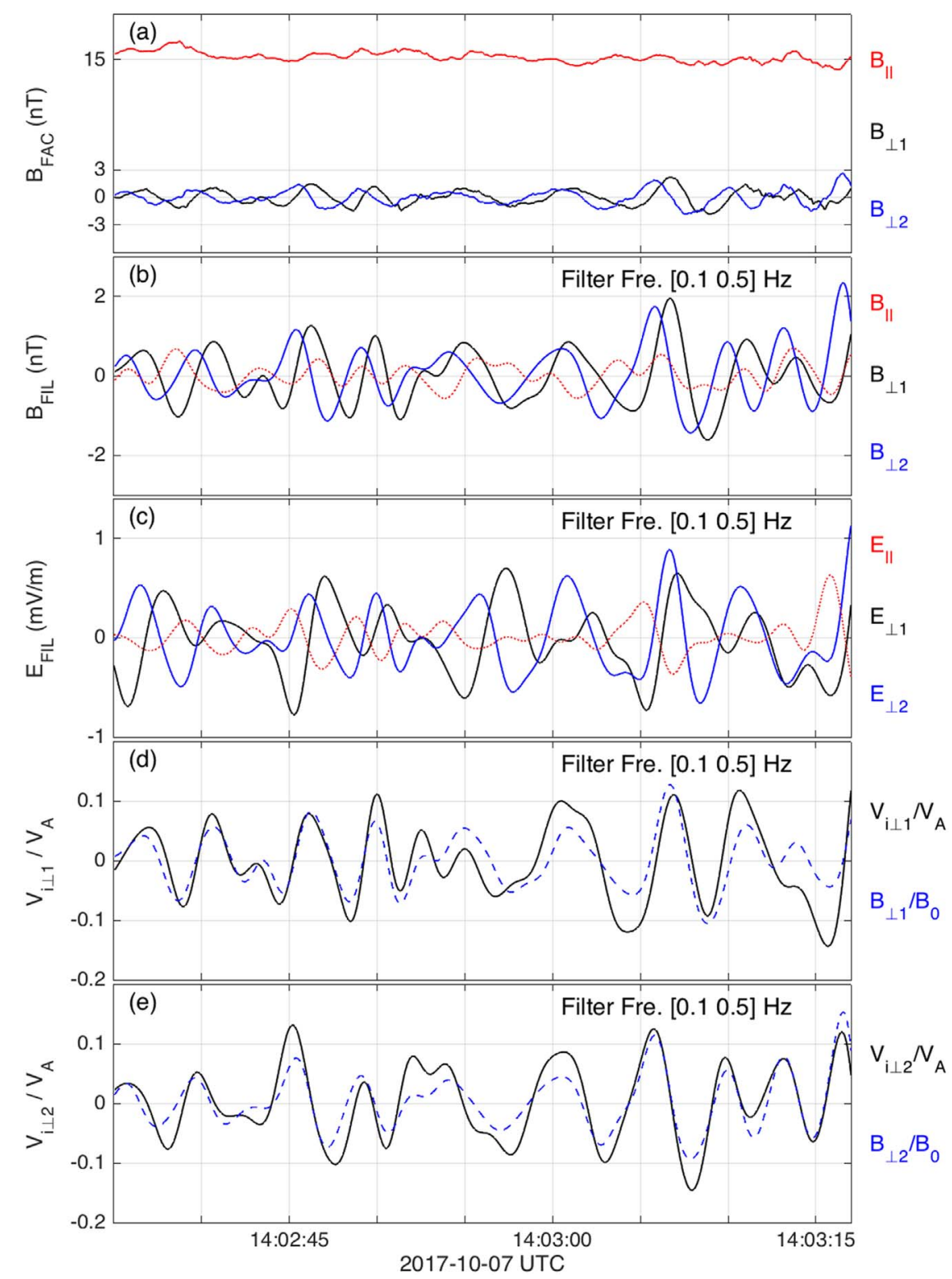

Figure 2. Electromagnetic and ion velocity fluctuations in magnetic field-aligned coordinates during 14:02:35.00UT-14:03:17.00UT. (a) Three magnetic field components, (b) magnetic fluctuations in the frequency range $0.1-0.5 \mathrm{~Hz}$, (c) electric fluctuations in $0.1-0.5 \mathrm{~Hz}$, (d), (e) ion velocities in units of Alfvén velocity in $0.1-0.5 \mathrm{~Hz}$. The magnetic fluctuations are overlaid in panels (d)-(e), labeled by the dashed lines.

reach $\sim 2 \mathrm{nT}$, where the ambient magnetic field is nearly $15 \mathrm{nT}$. The phase of $B_{\perp 1}$ is $\pi / 2$ ahead of $B_{\perp 2}$, indicating the left-hand polarization mode. Also, left-hand polarization is seen from the phase relation between $E_{\perp 1}$ and $E_{\perp 2}$ shown in panel (c); the considerable $B_{\|}$and $E_{\|}$arise. Panels (d) and (e) present the phase relations between magnetic and velocity components, that is, $V_{i \perp} / V_{A} \simeq B_{\perp} / B_{0}$, indicating the wave propagating antiparallel to the ambient magnetic field.

Figure 3 shows the ion energy distribution during the time interval from 14:02:35.00UT to 14:03:35.00UT. From panels (a), and (b), the ion omnidirectional flux mainly concentrates in the energy range $100 \mathrm{eV} \lesssim E_{i} \lesssim 1000 \mathrm{eV}$, and the ion pitch angle $\theta$ is normally larger than $90^{\circ}$. Panels (c) $-(\mathrm{g})$ give the ion pitch angle distribution (IPD) in different energy ranges. A chaotic IPD appears in the low-energy range $2.16 \mathrm{eV} \leqslant E_{i} \leqslant$ $58.89 \mathrm{eV}$, indicating very low ion density therein. It is interesting to see a periodic variation of IPD in the energy ranges $77.98 \mathrm{eV} \leqslant E_{i} \leqslant 239.63 \mathrm{eV}, 317.28 \mathrm{eV} \leqslant E_{i} \leqslant 736.45 \mathrm{eV}$, and
$975.08 \mathrm{eV} \leqslant E_{i} \leqslant 3967.62 \mathrm{eV}$. The change of pitch angle is nearly consistent with the variation of the angle $\theta_{B V_{i}}$ between the ion streaming velocity and magnetic field, defined as

$$
\theta_{B V_{i}}=\arccos \left(\frac{\boldsymbol{B} \cdot \boldsymbol{V}_{i}}{|B|\left|V_{i}\right|}\right)
$$

Moreover, both $\theta$ and $\theta_{B V_{i}}$ variations are closely related to the magnetic fluctuations shown in panel (i). Therefore, Figure 3 provides observational evidence for modulation of the ion pitch angle by large-amplitude cyclotron waves. For high-energy ions, $5253.24 \mathrm{eV} \leqslant E_{i} \leqslant 28301.89 \mathrm{eV}$, there is no modulation feature in the IPD.

Figure 4 shows the electron energy distribution during the time interval from 14:02:35.00UT to 14:03:35.00UT. From the omnidirectional electron differential energy flux (panel (a)), the electron energy is normally smaller than $1 \mathrm{keV}$. The electron pitch angle distribution (EPD) in panels (b)-(h) exhibits different 


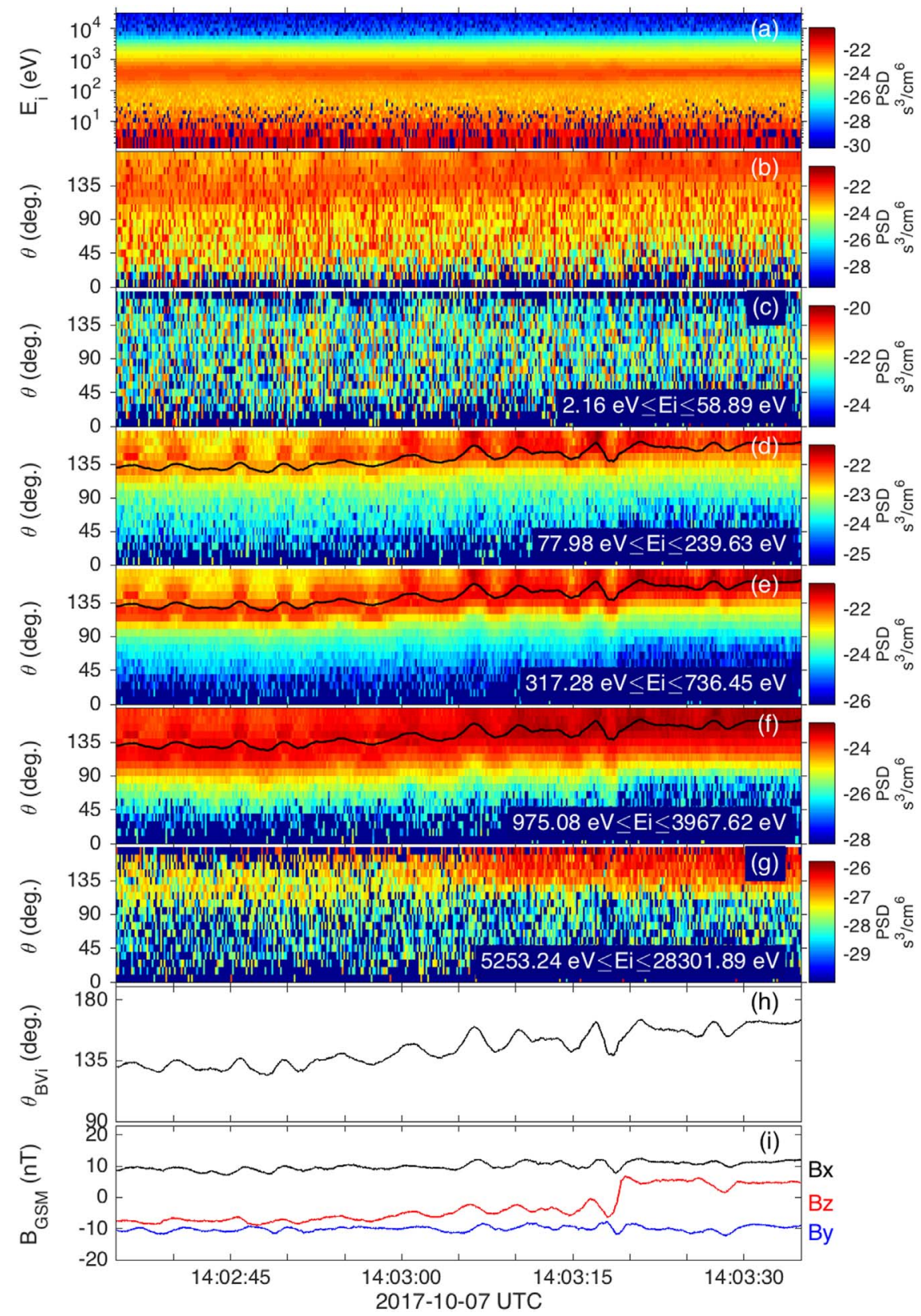

Figure 3. Ion dynamics in the time interval from 14:02:35.00UT to 14:03:35.00UT. (a) Omnidirectional ion differential energy flux, (b) ion pitch angle distribution (IPD) in the full energy range $2.16-28301.89 \mathrm{eV}$, (c)-(g) IPD in different energy ranges, (h) the angle $\theta_{B V_{i}}$ between the ion streaming velocity and magnetic field, and (i) magnetic field in Geocentric Solar Magnetospheric coordinates. $\theta_{B V_{i}}$ is overlaid in panels (d)-(f), labeled by black solid lines.

dynamics behaviors of electrons with different energy. For lowenergy electrons, $6.52 \mathrm{eV} \leqslant E_{e} \leqslant 19.15 \mathrm{eV}$, the pitch angle $\theta$ is always larger than $135^{\circ}$ before $t_{s \rightarrow n}$, and larger than $90^{\circ}$ after $t_{s \rightarrow n}$. This indicates low-energy electrons mainly streaming antiparallel to the magnetic field. In the energy range 25.07 $\mathrm{eV} \leqslant E_{e} \leqslant 42.95 \mathrm{eV}$, most electrons move along the magnetic field, i.e., $\theta \lesssim 45^{\circ}$. We also find the appearance of cooling for electrons at $\theta_{p}<\theta<180-\theta_{p}$, where the critical pitch angle $\theta_{p}$ is defined as $\theta_{p}=\arcsin \sqrt{B / B_{\max }}, B$ is the magnitude of the magnetic field, and $B_{\max }$ is the maximal $B$ during 14:02:35.00UT-14:03:35.00UT. Moreover, the cooling of trapped electrons arises in panel (e) where $56.23 \mathrm{eV} \leqslant$ $E_{e} \leqslant 73.60 \mathrm{eV}$. For $E_{e} \gtrsim 216.11 \mathrm{eV}$, trapped electrons are slightly heating, shown in panels $(\mathrm{g})$ and $(\mathrm{h})$. On the other hand, there exist many localized EPDs for electrons with $56.23 \mathrm{eV} \lesssim E_{e} \lesssim 830.63 \mathrm{eV}$. An example of localized EPD is shown as the band between the two vertical dashed lines in panels (e)-(g). The timescale of these localized EPDs approximates the period of the observed wave, implying the role of the largeamplitude cyclotron wave on the emergence of these confined electrons.

\section{Discussion and Summary}

Since the ion cyclotron anisotropic instability is widely believed to be the source of ion cyclotron waves in the Earth's magnetosphere (i.e., Cornwall 1965; Kennel \& Petschek 1966), here we check the possibility of local excitation of the observed 


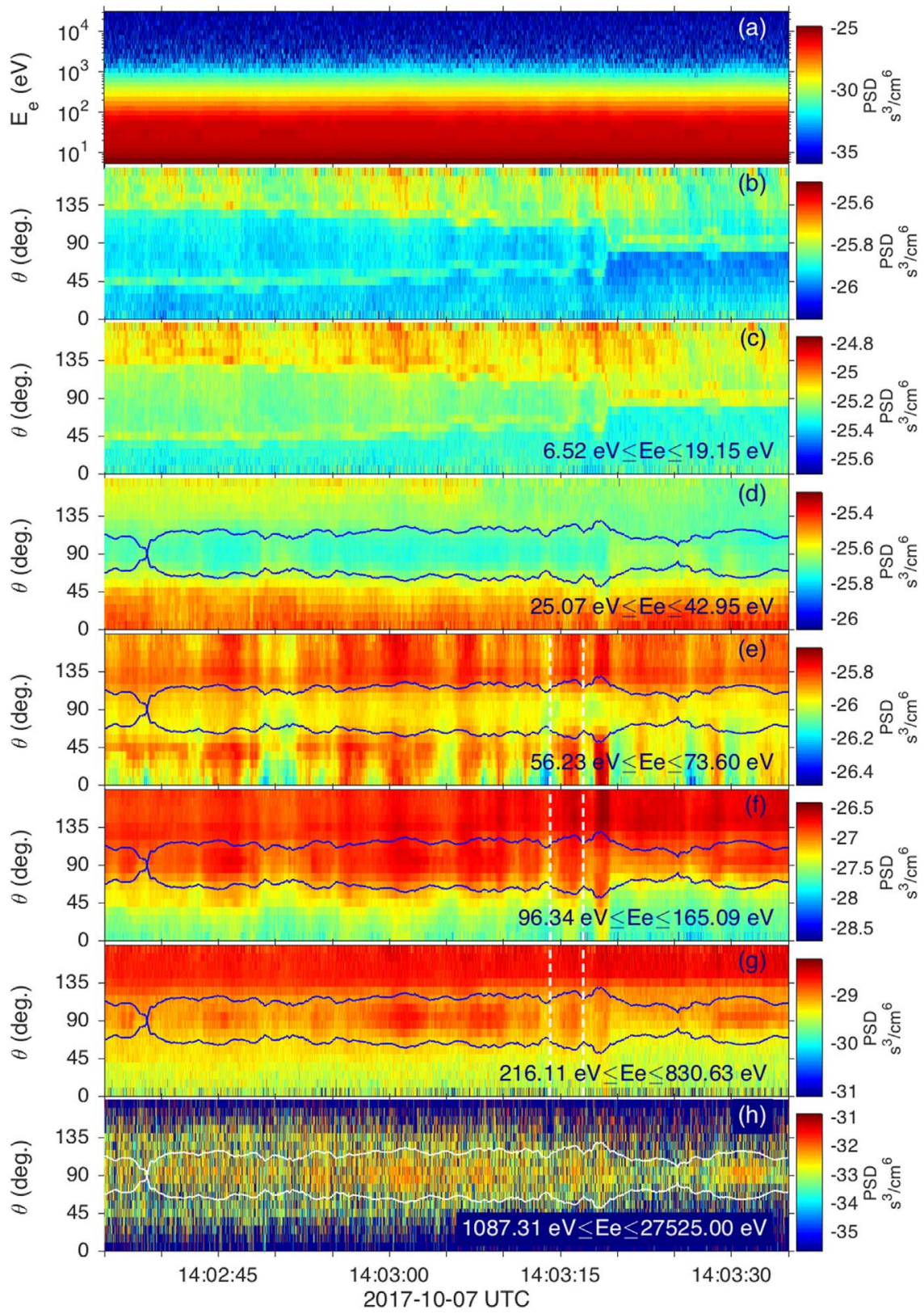

Figure 4. Electron dynamics during the time interval from 14:02:35.00UT to 14:03:35.00UT. (a) Omnidirectional electron differential energy flux, (b) electron pitch angle distribution (EPD) in the full energy range 6.52-27525.00 eV, and (c)-(h) EPD in different energy ranges. The solid lines overlaid in panels (d)-(h) represent critical pitch angles $\theta_{p}$ and $180^{\circ}-\theta_{p}$. An example of a localized EPD is shown as the band limited by the two vertical dashed lines in panels (e)-(g).

waves by this kind of instability. We adopt an instability threshold condition $\left(T_{i \perp} / T_{i \|}-1\right)=0.35 / \beta_{\|}^{0.42}$ (corresponding to $\left.\gamma / \Omega_{c i}=10^{-4}\right)$, assumed in plasmas only having electron and ion components (Gary \& Lee 1994). Using the plasma parameters averaged from FPI during 14:02:35UT-14:03:17UT, $n_{i}=12 \mathrm{~cm}^{-3}, T_{i \|}=91 \mathrm{eV}, T_{i \perp}=111 \mathrm{eV}, T_{e \|}=32 \mathrm{eV}, T_{e \perp}=$ $34 \mathrm{eV}$, and $B_{0}=15 \mathrm{nT}$, we find that $\left(T_{i \perp} / T_{i \|}-1\right)=$ $0.22<0.35 / \beta_{\|}^{0.42}=0.27$. Therefore, there is no ion temperature anisotropy instability in our event. Also, we use WHAMP (a dispersion equation solver based on linear plasma kinetic theory, Rönnmark 1982), and find that the wave is damping, i.e., $\gamma \sim-0.003 \Omega_{c i}$ at $\omega \sim 0.2 \Omega_{c i}$. On the other hand, Narita et al. (2004) found that some of the low-frequency, left-handpolarized Alfvén waves in the Earth's foreshock propagate downstream. Therefore, our observed waves may be generated nonlocally in the foreshock.

Since the observed waves propagate at $|\theta| \sim 0^{\circ}$, the wave frequency at the plasma frame is $\omega_{\text {plasma }} / \Omega_{c i} \approx$ $\left(\omega_{\text {obs }} / \Omega_{c i}\right)\left(1+V_{\|} / V_{A}\right)^{-1} \approx 0.15-0.6$, where the parallel ion streaming velocity is $V_{\|} \simeq 219 \mathrm{~km} \mathrm{~s}^{-1}$, the Alfvén velocity is $V_{A} \simeq 97 \mathrm{~km} \mathrm{~s}^{-1}$ during 14:02:35UT-14:03:17UT, and the observed wave frequency is $\omega_{\text {obs }} \sim 0.5 \Omega_{c i}-2 \Omega_{c i}$. From linear plasma theory we have linear responses among perpendicular electromagnetic components for the left-hand polarized cyclotron wave: $E_{\perp 2}=-i E_{\perp 1}, \quad B_{\perp 2}=-i B_{\perp 1}=\left(k_{\|} / \omega\right) E_{\perp 1}$, and $V_{i \perp 2}=-i V_{i \perp 1}=-\left(\omega / k_{\|}\right)\left(B_{\perp 2} / B_{0}\right) /\left(1-\omega / \Omega_{c i}\right)$ (i.e., Zhao 2015). When the wave is propagating anti-parallel to the ambient magnetic field, i.e., $k_{\|}$is negative, $E_{\perp 1}$ and $B_{\perp 2}$ are out 
of phase, $E_{\perp 2}$ and $B_{\perp 1}$ are in phase, and $V_{i \perp 1,2}$ and $B_{\perp 1,2}$ are in phase. These theoretical predictions are consistent with our observations. Furthermore, when the wave is obliquely propagating, even at a small normal angle, the parallel electromagnetic fields $E_{\|}$and $B_{\|}$will arise; for example, $E_{||} \sim 0.015 E_{\perp 1}$ and $B_{\|} \sim 0.16 B_{\perp 2}$ at $\omega \sim 0.2 \Omega_{c i}$ as $|\theta|=10^{\circ}$. Since the observed $E_{\|}$and $B_{\|}$, i.e., $E_{\|} \sim 0.2 E_{\perp}$ and $B_{\|} \sim 0.5 B_{\perp}$, are stronger than corresponding linear responses, this is in favor of $E_{||}$and $B_{\| \mid}$produced through the nonlinear mechanisms.

The most interesting finding in the study is the modulation of the ion and electron pitch angles by the observed largeamplitude electromagnetic cyclotron waves. The periodic variation of the pitch angle arises for ions with energy from $E_{i}=77.98 \mathrm{eV}$ to $E_{i}=3967.62 \mathrm{eV}$. When ions stream into the dusk-flank side of the magnetosheath, owing to large magnetic fluctuations, they induce a variation of the angle between the ion streaming velocity and the magnetic field, which results in the ion pitch angle having a periodic variation. On the other hand, some electrons will be trapped in the inhomogeneous magnetic field due to large magnetic fluctuations. Cooling occurs for trapped electrons with $25.07 \mathrm{eV} \lesssim E_{e} \lesssim 73.60 \mathrm{eV}$, and heating appears for trapped electrons with $E_{e} \gtrsim 216.11 \mathrm{eV}$. Moreover, for electrons having $56.23 \mathrm{eV} \lesssim E_{e} \lesssim 830.63 \mathrm{eV}$, their pitch angles exhibit an intermittent distribution feature, and the timescale is nearly the same as the wave period. This implies that electrons may be trapped by the large parallel electric field fluctuations. Therefore, the observed largeamplitude electromagnetic wave can affect both the dynamics of ions and electrons.

In summary, using $M M S$, this study observes the periodic variation of ion and electron pitch angles in the presence of large-amplitude, low-frequency, left-hand circularly polarized electromagnetic waves in the Earth's dusk-flank magnetosheath. Modulation of the ion pitch angle results from the change in the ion streaming velocity relative to the magnetic field including the ambient and fluctuation fields. Modulation of the electron pitch angle is induced by the inhomogeneous magnetic field structure, which is partly caused by the magnetic field fluctuation, and also probably by electrons trapped in the electromagnetic field of the large-amplitude wave.
This work was supported by the NNSFC 11673069, 41531071, by NSF of Jiangsu Province under grant No. BK20161617, and the Youth Innovation Promotion Association CAS. M.W.D. is supported by NNSFC 41874193 and 41574155, NERC grant NE/H004076/1, and by STFC in-house research grant. T.Y.W. is supported by the Marie Skłodowska-Curie grant No. 665593 from the European Union's Horizon 2020 research and innovation programme. J.S.H. is supported by NNSFC 41574168 and 41874200. The data are available from the $M M S$ Science Data Center (https://lasp.colorado.edu/mms/sdc/public/).

\section{ORCID iDs}

\section{J. S. Zhao (i) https://orcid.org/0000-0002-3859-6394}

\section{References}

Anderson, B. J., \& Fuselier, S. A. 1993, JGR, 98, 1461

Anderson, B. J., \& Fuselier, S. A. 1994, JGR, 99, 19413

Bortnik, J., Thorne, R. M., \& Omidi, N. 2010, JGR, 115, A12242

Burch, J. L., Moore, T. E., Torbert, R. B., \& Giles, B. L. 2016, SSRv, 199, 5 Cornwall, J. M. 1965, JGR, 70, 61

Dunlop, M. W., Lucek, E. A., Kistler, L. M., et al. 2002, JGRA, 107, 1228

Ergun, R. E., Tucker, S., Westfall, J., et al. 2016, SSRv, 199, 167

Gary, S. P., \& Lee, M. A. 1994, JGR, 99, 11297

Jian, L. K., Wei, H. Y., Russell, C. T., et al. 2014, ApJ, 786, 123

Jordanova, V. K., Albert, J., \& Miyoshi, Y. 2008, JGRA, 113, A00A10

Jordanova, V. K., Farrugia, C. J., Thorne, R. M., et al. 2001, JGR, 106, 7

Kennel, C. F., \& Petschek, H. E. 1966, JGR, 71, 1

Lindqvist, P.-A., Olsson, G., Torbert, R. B., et al. 2016, SSRv, 199, 137

Mauk, B. H., McIlwain, C. E., \& McPherron, R. L. 1981, GeoRL, 8, 103

Narita, Y., Glassmeier, K.-H., Schäfer, S., et al. 2004, AnGeo, 22, 2315

Omidi, N., Thorne, R. M., \& Bortnik, J. 2010, JGR, 115, A12241

Pollock, C. J., Moore, T., Jacques, A., et al. 2016, SSRv, 199, 331

Remya, B., Tsurutani, B. T., Reddy, R. V., et al. 2014, ApJ, 793, 6

Rönnmark, K. 1982, WHAMP-Waves in Homogeneous, Anisotropic, Multicomponent Plasmas, Tech. Rep. 179, Kiruna Geophysical Institute Roux, A., Perraut, S., Rauch, J. L., et al. 1982, JGR, 87, 8174

Russell, C. T., Anderson, B. R., Baumjohann, W., et al. 2016, SSRv, 199, 189 Summers, D., Ni, B., \& Meredith, N. P. 2007, JGRA, 112, A04206

Summers, D., \& Thorne, R. M. 2003, JGRA, 108, 1143

Tsurutani, B. T., Arballo, J. K., Zhou, X.-Y., Galvan, C., \& Chao, J. K. 2002, in COSPAR Coll. Ser., Space Weather Study Using Multipoint Techniques, ed. L.-H. Lyu (Oxford: Pergamon Press), 97

Wicks, R. T., Alexander, R. L., Stevens, M., et al. 2016, ApJ, 819, 6

Zhao, G. Q., Feng, H. Q., Wu, D. J., et al. 2018, JGRA, 123, 1715

Zhao, J. 2015, PhPl, 22, 042115

Zhu, H., Su, Z., Xiao, F., et al. 2012, JGR, 117, A12217 